\title{
Confirming the thermal Comptonization model for black hole X-ray emission in the low-hard state
}

\author{
M. Castro ${ }^{1}$, F. D'Amico ${ }^{1}$, J. Braga ${ }^{1}$, T. Maiolino ${ }^{2,3}$, K. Pottschmidt ${ }^{4}$, and J. Wilms ${ }^{5}$ \\ 1 Instituto Nacional de Pesquisas Espaciais (INPE), 12227-010 São José dos Campos, Brazil \\ e-mail: [castro; damico; braga]@das .inpe.br \\ 2 Physics Earth Sciences Department University of Ferrara, 44122 Ferrara, Italy \\ e-mail: maiolino@fe.infn.it \\ 3 Université de Nice Sophia-Antipolis, 06108 Nice, France \\ ${ }^{4}$ Cresst, NASA/GSFC \& UMBC, USA \\ e-mail: katja@milkyway.gsfc.nasa.gov \\ 5 Remeis Observatory \& ECAP, University of Erlangen-Nuremberg, 96049 Bamberg, Germany \\ e-mail: joern.wilms@sternwarte.uni-erlangen.de
}

Received 19 December 2013 / Accepted 16 July 2014

\section{ABSTRACT}

\begin{abstract}
Hard X-ray spectra of black hole binaries in the low/hard state are well modeled by thermal Comptonization of soft seed photons by a corona-type region with $k T \sim 50 \mathrm{keV}$ and optical depth around 1. Previous spectral studies of 1E 1740.7-2942, including both the soft and the hard X-ray bands, were always limited by gaps in the spectra or by a combination of observations with imaging and non-imaging instruments. In this study, we have used three rare nearly-simultaneous observations of 1E 1740.7-1942 by both $X M M$-Newton and INTEGRAL satellites to combine spectra from four different imaging instruments with no data gaps, and we successfully applied the Comptonization scenario to explain the broadband X-ray spectra of this source in the low/hard state. For two of the three observations, our analysis also shows that, models including Compton reflection can adequately fit the data, in agreement with previous reports. We show that the observations can also be modeled by a more detailed Comptonization scheme. Furthermore, we find the presence of an iron K-edge absorption feature in one occasion, which confirms what had been previously observed by Suzaku. Our broadband analysis of this limited sample shows a rich spectral variability in 1E 1740.7-2942 at the low/hard state, and we address the possible causes of these variations. More simultaneous soft/hard X-ray observations of this system and other black-hole binaries would be very helpful in constraining the Comptonization scenario and shedding more light on the physics of these systems.
\end{abstract}

Key words. radiation mechanisms: non-thermal - radiation mechanisms: thermal - X-rays: stars

\section{Introduction}

Since its discovery with the Einstein Observatory (Hertz \& Grindlay 1984), the putative black-hole system 1E 1740.7-2942 has been extensively studied. Historical observations (Skinner et al. 1987, 1991) have established 1E 1740.7-2942 as the brightest hard X-ray source $(E>20 \mathrm{keV})$ in the direction of the Galactic Center. From the years of observation with SIGMA/Granat, the source was discovered to have 3 spectral states resembling those of Cyg X-1 (Sunyaev et al. 1991). Another heritage from the SIGMA years was a detection of 1E 1740.7-2942 up to $\sim 500 \mathrm{keV}$ (Churazov et al. 1993). The source was dubbed a microquasar after radio jets were observed (Mirabel et al. 1992). The intense optical extinction toward the Galactic Center still prevents the identification of the counterpart of 1E 1740.7-2942 at optical and infrared wavelengths, despite all efforts (Del Santo et al. 2005; Smith et al. 2002; Martí et al. 2000). ASCA (Sakano et al. 1999) as well as Chandra (Gallo \& Fender 2002) that were helpful in determining the column density of hydrogen $\left(N_{\mathrm{H}}\right)$. Recent INTEGRAL observations have shown that the source can be clearly detected up to $\sim 600 \mathrm{keV}$ (Bouchet et al. 2009). A broadband study with Suzaku with a small spectral gap (10-12 keV) has shown the presence for the first time of an iron K-edge absorption in the 1E 1740.7-2942 spectra (Reynolds \& Miller 2010).
In general, the combined soft/hard X-ray spectra of 1E 1740.7-2942 are fitted by a combination of a thermal component and a non-thermal powerlaw. These fits are useful in constraining the spectral state of 1E 1740.7-2942 through the powerlaw index (see, e.g., Remillard \& McClintock 2006). Alternatively, a combination of two thermal models can be used to explain the broadband spectrum of this source: a soft component, which is associated with the accretion disk, and a hard Comptonized component coming from a corona-type region which has $k T \sim 50 \mathrm{keV}$ and an optical depth around 1 (e.g., Bouchet et al. 2009). In this study, we show evidence that modeling the spectra of 1E 1740.7-2942 in the latter way provides a consistent picture. We also report a detection here (by XMM) of an iron K-edge absorption feature, which confirms the Suzaku results (Reynolds \& Miller 2010).

Recently, a NuSTAR + INTEGRAL study on 1E 1740.72942 was published based on data of 2012 (Natalucci et al. 2014). The spectrum, starting at $\sim 3 \mathrm{keV}$ and extending up to $250 \mathrm{keV}$, was fitted by a combination of a Comptonization model (compTT) and a soft component (diskbb), which enables us to make useful comparisons with our results. Finally, we highlight that this present study is an extended and improved version of a previous work, where we analyzed data for only two epochs (2003 and 2005) and only from PN/XMM and IBIS/ INTEGRAL (Castro et al. 2012). 
A\&A 569, A82 (2014)

Table 1. XMM and INTEGRAL simultaneous observations of 1E 1740.7-2942.

\begin{tabular}{|c|c|c|c|c|c|}
\hline & & $\mathrm{PN}$ & MOS1 & JEMX & ISGRI \\
\hline \multirow{2}{*}{2003} & $\operatorname{TStart}(\mathrm{UTC})^{(a)}$ & 2003-09-11 23:05:36 & 2003-09-11 23:00:21 & 2003-09-11 22:49:29 & $2003-09-1122: 50: 31$ \\
\hline & Exposure(ks) & 1.6 & 8.0 & 3.0 & 9.0 \\
\hline \multirow{2}{*}{2005} & TStart(UTC) & 2005-10-02 01:21:18 & 2005-10-02 01:16:05 & 2005-10-02 11:07:19 & $2005-10-02$ 02:48:43 \\
\hline & Exposure(ks) & 16.0 & 7.7 & 2.2 & 23.4 \\
\hline \multirow{2}{*}{2012} & TStart(UTC) & 2012-04-03 08:32:12 & 2012-04-03 08:27:03 & 2012-04-07 09:01:18 & 2012-04-07 05:59:52 \\
\hline & Exposure(ks) & 82.7 & 34.1 & 1.6 & 7.7 \\
\hline
\end{tabular}

Notes. ${ }^{(a)}$ Time expressed in yyyy-mm-dd hh:mm:ss as derived from each produced spectrum file.

\section{Data selection and analysis}

To test the thermal Comptonization model in the low/hard state of 1E 1740.7-2942, we carried out a search in the databases of XMM and INTEGRAL and looked for nearly simultaneous observations. The best matches satisfying our criteria are presented in Table 1, which results in three observations. The 2003 observation was performed by the two satellites almost simultaneously. Some hours of delay can be seen in the 2005 data set, and in 2012 the data from the two satellites are not simultaneous with days of delay in between the observations.

Data from XMM-Newton (Jansen et al. 2001) cameras PN (Strüder et al. 2001), and MOS1 (Turner et al. 2001) were reduced using standard procedures with SAS (V. 12.0.1) ${ }^{1}$. INTEGRAL (Winkler et al. 2003) data from IBIS (Ubertini et al. 2003) and JEM-X (Lund et al. 2003) telescopes were treated using the recipes described in the OSA 10.0 documentation ${ }^{2}$. We have also made use of XSPEC (V. 12.8.0) in performing our spectral fits. Data from PN were constrained to the $\sim 2$ to $12 \mathrm{keV}$ region, and MOS1 data were limited to the $\sim 2-10 \mathrm{keV}$ band. The lower energy threshold is due to the low count rate and signal-to-noise ratio $(\mathrm{S} / \mathrm{N})$ below $\sim 2 \mathrm{keV}$ for both cameras (PN and MOS1) according to the pileup analysis. We also note that $\sim 2 \mathrm{keV}$ was the lower limit in energy used by ASCA (Sakano et al. 1999) and Chandra on two occasions (Cui et al. 2001; Gallo \& Fender 2002). With our spectra also starting around $2 \mathrm{keV}$ we left the hydrogen column density as a free parameter in our fits.

The energy range from $\sim 10$ up to $\sim 20 \mathrm{keV}$ was filled with the use of the JEM-X/INTEGRAL telescope, even though the 1E 1740.7-2942 count rates are quite low in this band for this instrument. The data presented here from 20 up to $200 \mathrm{keV}$ were collected by the IBIS telescope onboard INTEGRAL, completing our broadband coverage. We have made use only of the ISGRI/IBIS data (Lebrun et al. 2003).

Fits to our spectra included the multiplicative const and phabs components in XSPEC when one accounts for the difference in the counts for the four instruments and the other for absorption by neutral material. The normalization factors were relative to the PN instrument, which has the highest count rate among the four instruments.

While the ASCA (Sakano et al. 1999) and Chandra (Gallo $\&$ Fender 2002) studies on 1E 1740.7-2942 have made use of an absorbed powerlaw to fit the $2-20 \mathrm{keV}$ spectra, the broadband (2-200 keV) Suzaku study (Reynolds \& Miller 2010) used a combination of models. On the other hand, the INTEGRAL

\footnotetext{
1 http://xmm.esa.int/sas/

2 http://www.isdc.unige.ch/integral/
}

hard X-ray spectrum of 1E 1740.7-2942 has been fitted with a Comptonization model (Bouchet et al. 2009).

We have thus fitted our XMM and INTEGRAL broadband (2-200 keV) spectra of 1E 1740.7-2942 with two components: a soft standard extended blackbody (diskbb), which comes from the accretion disk and a Comptonized component. We have made use of thermal Comptonization and other variations. This component is probably coming from a larger region (a corona). In this two-component model, we tied (as usual) the temperature of the seed soft photons of the Comptonized component to the disk average temperature (modeled as diskbb). To help us in classifying the 1E 1740.7-2942 spectral state, we have also made use of a classical exponential folded powerlaw to fit our spectra (see details in Remillard \& McClintock 2006). We note that this model had also been used before for 1E 1740.7-2942 (e.g., Bouchet et al. 2009).

\section{Results}

The lower (XMM) parts of our spectra can be fitted very well as an accretion disk consisting of multiple blackbody components, as the diskbb model. It is noteworthy, however, that another (soft) component is necessary $\left(F_{\text {test }}\right.$ of the order of $10^{-7}$ ) to adequately describe the soft part or our 1E 1740.7-2942 data in 2003 and 2012. This component is only marginally needed in the 2005 spectrum $\left(F_{\text {test }}\right.$ of $\left.10^{-4}\right)$. Nevertheless, to maintain uniformity in our comparisons between the three spectra, we kept this second component in the XMM spectrum of 2005. This second component is very well fitted by any Comptonization model. With the addition of this Comptonization component, our broadband 2-200 keV spectra can be very well modeled. As we have already stated, instead of using Comptonization models, an exponential folded powerlaw can also be used to fit (the INTEGRAL) part of our spectra. We also performed those fits accordingly, which help us to conclude (see details in Remillard $\&$ McClintock 2006) that 1E 1740.7-2942 was in its (canonical) low/hard state (LHS) in the three observations reported in this study.

It is interesting to note that we found no evidence in any of our XMM data sets of the emission lines due to the soft X-ray background in the direction of this source, as reported, for example, in Reynolds \& Miller (2010). A feature near $7 \mathrm{keV}$ was found, however. Even though it was apparent from the PN spectrum that the feature seems to be an edge, the feature is very well fitted by a Gauss model in XSPEC. The centroid energy is $7.11 \mathrm{keV}$, which is exactly the energy of the iron absorption K-edge reported by Suzaku. We proceed as in the Suzaku study, modeling this edge using the zvfeabs model in XSPEC. The 


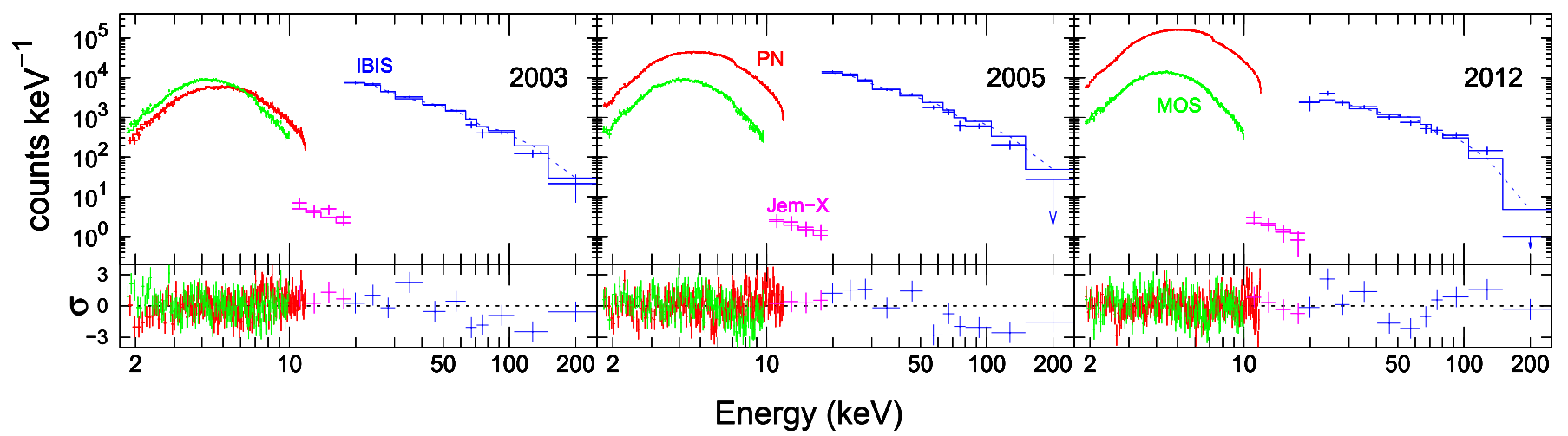

Fig. 1. XMM and INTEGRAL spectra of 1E 1740.7-2942 for 2003, 2005, and 2012 observations described in this study for a fitted diskbb + compTT model. In the 2012 spectrum a Fe-edge in $7.1 \mathrm{keV}$ (modeled as Gauss line: see text) is also present. Instruments are labeled in upper panels, which also shows the data, while bottom panels correspond to the residuals of the fits. In the middle and right upper panels, the bins at $\sim 200 \mathrm{keV}$ are $3 \sigma$ upper limits. In all the three upper panels, a dotted blue line shows the relative contribution of the compTT component in the ISGRI part of the spectrum. Fit parameters can be found in Table 2.

fit, in this case, returns an iron absorption K-edge of $7.19 \mathrm{keV}$. Freezing the edge energy to 7.11 returned a $\chi_{\text {red }}^{2}$ value of 1.4 , which is not as good as the value obtained with the Gauss model (1.2). Notwithstanding this difference in goodness of fit, it is our interpretation on physical grounds that this is the iron absorption K-edge observed before by Suzaku (Reynolds \& Miller 2010). To our knowledge, this is the first detection by XMM of this feature in 1E 1740.7-2942.

For the Comptonization component, our first attempts were with the simplest thermal compTT (Titarchuk 1994) model in $X S P E C$, since this form of modeling was widely used in past spectra modeling of 1E 1740.7-2942, and for others black hole binaries and in a previous version of this study (Castro et al. 2012). The spectra resulting of those fits are shown in Fig. 1.

All the fits with this thermal Comptonization provided a very adequate description of the spectrum. In our fits we kept the geometry parameter equal to 1 , which corresponds to a disk geometry in compTT. Fits parameters can be found in Table 2.

Motivated by the evidence of Compton reflection found by some authors in 1E 1740.7-2942 (Del Santo et al. 2005), and by the study of Natalucci et al. (2014), which found no evidence of it, we also used the convolutive reflect component in our fits (Magdziarz \& Zdziarski 1995). Presence of reflection is very common in the low/hard spectra of black hole binaries (see, e.g., Zdziarski et al. 1999). If the Comptonizing plasma is surrounding the disk, then the presence of Compton reflection is unavoidable. The results of our first attempt in modeling such reflection, with the reflect model (acting on the compTT component) is shown in Table 2. We found indications of the possible presence of the Compton reflection in the 2003 and 2005 spectra but not in the 2012 spectrum. It is interesting to note that the absence of reflection in 2012 and the presence of an Fe-edge agrees with the results of Reynolds \& Miller (2010). As can be see in Table 2, the presence or absence of the Compton reflection does not alter the parameters of the fit with respect to the compTT fit alone (i.e., without the convolutive reflect component).

We have also made an attempt to fit our spectra with the compPS model (Poutanen \& Svensson 1996) in XSPEC to check what has been tried before (Del Santo et al. 2005). CompPS provides a numerical solution of the radiative transfer equation, and it comprises Compton reflection as one of its parameters. However, we noted that the 2012 spectrum in our fits did not fit very well by this model in the sense that it returns an unrealistic value for the plasma temperature when using a slab geometry

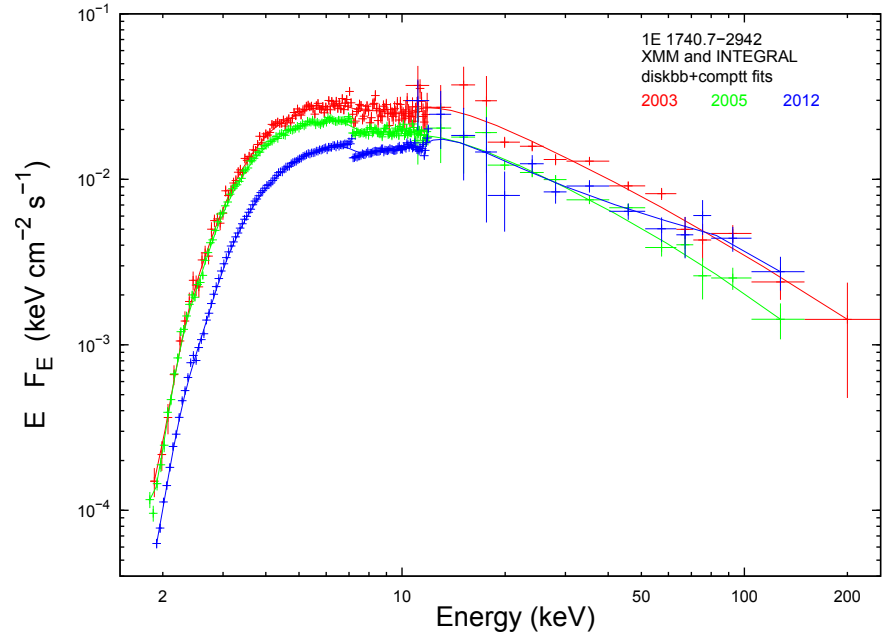

Fig. 2. Absorbed spectra of 1E 1740.7-2942, which are modeled with diskbb + compTT, for the three XMM + INTEGRAL observations shown in this study. In only the 2012 spectrum, a feature at $7.11 \mathrm{keV}$ (modeled as a Gauss in XSPEC), interpreted as being a Fe-edge (see text for details) is also included.

with an optical depth around the value of one, as is the case for our 2003 and 2005 fits with compTT. Therefore, we decided not to use the compPS model in this study.

In Fig. 2, we show the spectral variation between the 2003, 2005, and 2012 spectrum.

We also show the parameters of our fits to a exponential folded powerlaw model (cutoffpl in XSPEC) in Table 2.

In Table 3, we show the measured fluxes for the observations in this study. It is interesting to compare our broadband fluxes with those obtained by Suzaku (Reynolds \& Miller 2010). The average flux measured by Suzaku in two observations (one in 2006 and another in 2008) is $2.2 \times 10^{-9} \mathrm{erg} \mathrm{cm}^{-2} \mathrm{~s}^{-1}$ in the $2-300 \mathrm{keV}$ range. For our observations, the average between 2003 and 2005 observations is $2.5 \pm 0.4 \times 10^{-9} \mathrm{erg} \mathrm{cm}^{-2} \mathrm{~s}^{-1}$ in the same energy range $\left(2.97 \pm 0.1 \times 10^{-9}\right.$ in 2003 and $1.97 \pm 0.2 \times 10^{-9}$ in 2005 , in erg $\mathrm{cm}^{-2} \mathrm{~s}^{-1}$ ), whereas our 2012 value is $1.7 \pm$ $0.1 \times 10^{-9} \mathrm{erg} \mathrm{cm}^{-2} \mathrm{~s}^{-1}$ (unabsorbed fluxes), characterizing a significant decrease in the $2-300 \mathrm{keV}$ flux from $1 \mathrm{E} 1740.7-2942$ from 2003 up to 2012. 
A\&A 569, A82 (2014)

Table 2. Model parameters of fitting XMM and INTEGRAL 1E 1740.7-2942 spectra.

\begin{tabular}{|c|c|c|c|c|}
\hline \multicolumn{5}{|c|}{ diskbb + compTT } \\
\hline & & 2003 & 2005 & 2012 \\
\hline phabs & $N_{\mathrm{H}}\left(10^{22} \mathrm{~cm}^{-1}\right)$ & $14.1_{-0.3}^{+0.3}$ & $12.5_{-0.1}^{+0.1}$ & $14.1_{-0.1}^{+0.1}$ \\
\hline diskbb & $T_{\text {in }}(\mathrm{keV})$ & $0.24_{-0.02}^{+0.02}$ & $0.17_{-0.03}^{+0.03}$ & $0.19_{-0.01}^{+0.01}$ \\
\hline \multirow{3}{*}{ compTT } & $T_{0}(\mathrm{keV})$ & $=T_{\text {in }}$ & $=T_{\text {in }}$ & $=T_{\text {in }}$ \\
\hline & $k T(\mathrm{keV})$ & $65.6_{-1.9}^{+2.2}$ & $65.7_{-1.9}^{+1.5}$ & $20.1_{-0.1}^{+0.1}$ \\
\hline & $\tau$ & $0.90_{-0.02}^{+0.08}$ & $0.85_{-0.02}^{+0.02}$ & $3.56_{-0.03}^{+0.03}$ \\
\hline Gauss $^{(a)}$ & LineE $(\mathrm{keV})$ & - & - & $7.11_{-0.01}^{+0.01}$ \\
\hline$\chi^{2} /$ d.o.f. & & $308 / 277$ & $400 / 294$ & $352 / 298$ \\
\hline \multicolumn{5}{|c|}{ diskbb + reflect*compTT } \\
\hline phabs & $N_{\mathrm{H}}\left(10^{22} \mathrm{~cm}^{-2}\right)$ & $13.4_{-0.3}^{+0.4}$ & $12.5_{-0.1}^{+0.1}$ & $14.0_{-0.1}^{+0.1}$ \\
\hline diskbb & $T_{\mathrm{in}}(\mathrm{keV})$ & $0.21_{-0.03}^{+0.03}$ & $0.16_{-0.01}^{+0.01}$ & $0.17_{-0.01}^{+0.01}$ \\
\hline reflect & $\Omega / 2 \pi$ & $0.74_{-0.28}^{+0.29}$ & $0.32_{-0.14}^{+0.15}$ & $\leq 0.01^{(b)}$ \\
\hline \multirow{4}{*}{ compTT } & $T_{0}$ & $=T_{\mathrm{in}}$ & $=T_{\text {in }}$ & $=T_{\text {in }}$ \\
\hline & $k T(\mathrm{keV})$ & $64.7_{-2.4}^{+2.6}$ & $65.5_{-2.0}^{+2.1}$ & $20.8_{-0.4}^{+0.4}$ \\
\hline & $\tau$ & $0.88_{-0.05}^{+0.05}$ & $0.80_{-0.03}^{+0.03}$ & $3.52_{-0.03}^{+0.03}$ \\
\hline & norm & $44_{-3}^{+3} \times 10^{-4}$ & $43_{-5}^{+7} \times 10^{-4}$ & $76_{-2}^{+2} \times 10^{-4}$ \\
\hline Gauss $^{(a)}$ & LineE (keV) & - & - & $7.11_{-0.01}^{+0.01}$ \\
\hline$\chi^{2} /$ d.o.f. & & $296 / 275$ & $394 / 292$ & $347 / 295$ \\
\hline \multicolumn{5}{|c|}{ diskbb + cutoffpl } \\
\hline phabs & $N_{\mathrm{H}}\left(10^{22} \mathrm{~cm}^{-2}\right)$ & $13.0_{-0.3}^{+0.4}$ & $12.3_{-0.1}^{+0.1}$ & $13.8_{-0.1}^{+0.1}$ \\
\hline diskbb & $T_{\mathrm{in}}(\mathrm{keV})$ & $0.21_{-0.03}^{+0.03}$ & $0.15_{-0.04}^{+0.04}$ & $0.17_{-0.01}^{+0.01}$ \\
\hline \multirow{3}{*}{ cutoffpl } & $\Gamma$ & $1.42_{-0.04}^{+0.04}$ & $1.52_{-0.02}^{+0.02}$ & $1.24_{-0.02}^{+0.02}$ \\
\hline & $E_{\text {cut }}(\mathrm{keV})$ & $89.7_{-12.1}^{+15.7}$ & $87.1_{-11.4}^{+14.5}$ & $138_{-30.1}^{+51.7}$ \\
\hline & norm & $85_{-6}^{+7} \times 10^{-3}$ & $81_{-3}^{+3} \times 10^{-3}$ & $333_{-8}^{+8} \times 10^{-4}$ \\
\hline Gauss $^{(a)}$ & LineE $(\mathrm{keV})$ & - & - & $7.11_{-0.01}^{+0.01}$ \\
\hline$\chi^{2} /$ d.o.f. & & $286 / 276$ & $379 / 293$ & $350 / 296$ \\
\hline
\end{tabular}

Notes. Quoted errors are $1 \sigma .{ }^{(a)}$ This (additive) component is necessary to fit the 2012 spectrum (see text for details). ${ }^{(b)} 3 \sigma$ upper limit in the parameter.

Table 3. XMM and INTEGRAL measured (absorbed) fluxes for 1E 1740.7-2942 in erg $\mathrm{cm}^{-2} \mathrm{~s}^{-1}$.

\begin{tabular}{cccccc}
\hline \hline & & $2-10 \mathrm{keV}$ & $10-20 \mathrm{keV}$ & $20-50 \mathrm{keV}$ & $50-200 \mathrm{keV}$ \\
\cline { 2 - 6 } 2003 & diskbb & $1.15_{-0.06}^{+0.06} \times 10^{-12}$ & 0 & 0 & 0 \\
& compTT & $2.74_{-0.14}^{+0.14} \times 10^{-10}$ & $3.72_{-0.19}^{+0.19} \times 10^{-10}$ & $5.65_{0.28}^{+0.28} \times 10^{-10}$ & $1.02_{-0.05}^{+0.05} \times 10^{-9}$ \\
& total & $2.76_{-0.14}^{+0.14} \times 10^{-10}$ & $3.72_{-0.19}^{+0.19} \times 10^{-10}$ & $5.65_{-0.28}^{+0.28} \times 10^{-10}$ & $1.02_{-0.05}^{+0.05} \times 10^{-9}$ \\
\hline \multirow{2}{*}{2005} & diskbb & $1.18_{-0.20}^{+0.20} \times 10^{-13}$ & 0 & 0 & 0 \\
& compTT & $2.57_{-0.26}^{+0.28} \times 10^{-10}$ & $2.56_{-0.26}^{+0.28} \times 10^{-10}$ & $3.77_{-0.38}^{+0.41} \times 10^{-10}$ & $6.44_{-0.64}^{+0.71} \times 10^{-10}$ \\
& total & $2.57_{-0.26}^{+0.28} \times 10^{-10}$ & $2.56_{-0.26}^{+0.28} \times 10^{-10}$ & $3.77_{-0.38}^{+0.41} \times 10^{-10}$ & $6.44_{-0.64}^{+0.71} \times 10^{-10}$ \\
\hline \multirow{2}{*}{2012} & diskbb & $3.16_{-0.01}^{+0.02} \times 10^{-13}$ & 0 & 0 & 0 \\
& compTT & $1.86_{-0.01}^{+0.02} \times 10^{-10}$ & $2.76_{-0.01}^{+0.01} \times 10^{-10}$ & $3.89_{-0.01}^{+0.02} \times 10^{-10}$ & $6.11_{-0.01}^{+0.01} \times 10^{-10}$ \\
& total & $1.86_{-0.01}^{+0.02} \times 10^{-10}$ & $2.76_{-0.01}^{+0.01} \times 10^{-10}$ & $3.89_{-0.01}^{+0.02} \times 10^{-10}$ & $6.11_{-0.01}^{+0.01} \times 10^{-10}$ \\
\hline
\end{tabular}

Notes. Errors are $1 \sigma$.

It is also interesting to compare our results for the hydrogen column density $\left(N_{\mathrm{H}}\right)$ with other studies. Observations with ASCA (Sakano et al. 1999) found an average value of $9.7 \pm 0.4$ in eight observations. A Chandra/HETGS study found $11.8 \pm 0.6$
(Cui et al. 2001), whereas a Chandra/ACIS-I found $10.5 \pm 0.6$ (Gallo \& Fender 2002). The average in our three observations is $13.6 \pm 0.1$. The average of all of these results is $13.3 \pm 0.1$. Without our results, the average is $10.4 \pm 0.3$ with all quoted 
values in units of $10^{22} \mathrm{~cm}^{-2}$. We caution the reader that we performed our fits leaving $N_{\mathrm{H}}$ as a free parameter. We then carefully verified that neither our conclusions nor the quality of our fits is changed noticeably by adopting (and freezing) $N_{\mathrm{H}}$ to 10.4 .

\section{Discussion}

The observations we describe in this study are one of the few with broadband (2-200 keV) coverage of 1E 1740.7-2942 spectra with no data gaps, which is provided only by imaging instruments, avoiding any source confusion and, thus, flux contamination. In our study, we tested the thermal-Compton paradigm for the source spectra by following, for example, other studies of 1E 1740.7-2942 spectra. Such a paradigm was first tested in Cyg X-1 spectral analysis (Gierlinski et al. 1997).

For the first time to our knowledge, we reported here evidence of a $7.11 \mathrm{keV}$ Fe-edge detected by XMM, which agrees with previous reports for such a feature derived from Suzaku studies (Reynolds \& Miller 2010).

Our results for the magnitude of $N_{\mathrm{H}}$ for 1E 1740.7-2942 show a higher value than the average. However, we do not claim here a variation of $N_{\mathrm{H}}$ in the line of sight of 1E 1740.7-2942, as well as any intrinsic change at or nearby the source, since our fits are not very sensitive to the value of $N_{\mathrm{H}}$.

Our model of 1E 1740.7-2942 spectra shows a variation in the optical depth of the plasma between 2012 when compared to 2003 or 2005 . This variation implies a change in the so-called $y$ parameter of inverse Compton scattering (see, e.g., Rybicki \& Lightman 1986) on the order of 3. From our database, it is not clear what causes this variation. More simultaneous observations by XMM and INTEGRAL will be very important in shedding light in this issue.

Compared to the Suzaku study of 1E 1740.7-2942 (Reynolds \& Miller 2010), we verified a possible decrease in the $2-300 \mathrm{keV}$ unabsorbed flux of the source. Our average value found for 2003 and 2005 is $2.5 \pm 0.4 \times 10^{-9} \mathrm{erg} \mathrm{cm}^{-2} \mathrm{~s}^{-1}$, and our measured value for 2012 is $1.7 \pm 0.1 \times 10^{-9} \mathrm{erg} \mathrm{cm}^{-2} \mathrm{~s}^{-1}$, while the averaged between 2006 and 2008 value measured by Suzaku is $2.2 \times 10^{-9} \mathrm{erg} \mathrm{cm}^{-2} \mathrm{~s}^{-1}$. It is noteworthy that recent monitoring by the INTEGRAL/IBIS program in the Galactic Bulge reported that 1E 1740.7-2942 is below the detection sensitivity limit (Kuulkers et al. 2013).

From the results in Table 3, we can clearly see a decrease in the 50-200 keV flux. The absorbed flux in the $2-10 \mathrm{keV}$ range remained within errors constant in 2003 and 2005 and then decreased in 2012. In trying to associate the change in the 50-200 keV flux with some parameter of our models, no clear evidence is found, i.e., our decrease in flux is not evidently correlated with any parameter. This is contrary to what was observed in GX 339-4, where a decrease in the luminosity is associated with an increase in $k T_{\mathrm{e}}$ (Wardziński et al. 2002). The high energy 50-200 keV flux must be associated with the accretion disk (or, for example, with a corona surrounding it), and, similarly to other Galactic black holes, it is the brightest component of the X-ray spectrum when the source is in the LHS.

The comparison of our derived plasma temperatures with other broadband hard X-ray studies of 1E 1740.7-2942 must be considered with caution. For example, our measured temperatures are different from the ones reported by Del Santo et al. (2005), but this is probably due to the fact that these authors have used the physical assumptions of the compPS model, which was not used here. Similarly, a different modeling was used by Bouchet et al. (2009) and Reynolds \& Miller (2010). On the other hand, low temperatures of $\sim 20 \mathrm{keV}$, as in our models of the 2012 spectrum, were already reported, for instance, by the NuSTAR recent study (Natalucci et al. 2014), which has made use of the same models we applied (i.e., diskbb and compTT). We caution the reader that the NuSTAR reported value for the optical depth is of the order of 1.4, while it varies in our observations (see Table 2).

It is also noteworthy that our fits with the cutoffpl model also provided acceptable fits, implying that non-thermal process may also explain the broadband 1E 1740.7-2942 behavior. We note that a model based on a jet emission for explaining the hard X-ray spectrum of 1E 1740.7-2942 was ruled out (Bosch-Ramon et al. 2006). A model consisting of two thermal Comptonization components was already used (Bouchet et al. 2009) as an alternative to non-thermal processes.

In our spectral analysis for 2003 and 2005, we found the presence of Compton reflection, which agrees with previous reports for 1E 1740.7-2942 spectra (e.g., Del Santo et al. 2005). Our 2012 spectral modeling, however, found no evidence for such a component, which is also compatible with other results (e.g., Natalucci et al. 2014). It is very interesting to note that in 2012 the observation of a Fe-edge in our spectrum without the presence of Compton reflection is also agrees with previous spectral analysis of 1E 1740.7-2942 (Reynolds \& Miller 2010). Since the Compton reflection seems not to be permanent in 1E 1740.7-2942 spectra, it is tempting to associate the vanishing of such a feature with physical changes in possible corona surrounding the accretion disk.

Our study has revealed a rich spectral variability in 1E 1740.7-2942 by highlighting the importance of broadband coverage by XMM and INTEGRAL in future simultaneous observations.

\section{Conclusions}

We have shown a simultaneous broadband study of 1E 1740.7-2942 in three different epochs with the use of ESA's XMM and INTEGRAL satellites here that covers the band from $\sim 2$ up to $200 \mathrm{keV}$ with no data gaps. The imaging instruments onboard XMM and INTEGRAL prevent any kind of source-confusion/flux contamination. To our knowledge for the first time, we reported here a XMM/PN observation of the iron absorption K-edge at $7.11 \mathrm{keV}$, a value reported previously in Suzaku studies. We derived an historical decrease in the 2-300 keV flux of 1E 1740.7-2942.

Our study has revealed a rich spectral variability in 1E 1740.7-2942. We have shown that the plasma temperature has varied between the 2003-2005 and the 2012 spectra, but it is unclear from our analysis what the causes of this are. We note that this variation is accompanied by a huge increase in the optical depth from 2003 to 2012.

We believe that only more broadband observations of 1E 1740.7-2942, for example, with simultaneous XMM and INTEGRAL campaigns as the ones discussed in this study, will be able to provide a data base from where tight constrains can be derived to the Comptonization model for the emission in the LHS state of 1E 1740.7-2942.

Acknowledgements. M.C. gratefully acknowledges CAPES/Brazil for support. M.C. gratefully acknowledges Mariano Méndez at the Kapteyn Astronomical Institute (Groningen-The Netherlands) host in a visit in the period of September - October/2012, sponsored by the COSPAR Program for Capacity Building Fellowship, for helpful discussions in the subject of this study. M.C. and F.D. acknowledges Raimundo Lopes de Oliveira Filho for early guidance in XMM data analysis issues. F.D. acknowledges Tomaso Belloni for helpful discussions. We deeply acknowledge Andrzej Zdziarski, our referee, for superb comments and for helping us in improving the quality of this study. 


\section{References}

Bosch-Ramon, V., Romero, G. E., Paredes, J. M., et al. 2006, A\&A, 457, 1011 Bouchet, L., del Santo, M., Jourdain, E., et al. 2009, ApJ, 693, 1871

Castro, M. A., Maiolino, T., D’Amico, F., et al. 2012, PoS (Integral 2012)046

Churazov, E., Gilfanov, M., Sunyaev, R., et al. 1993, ApJ, 407, 752

Cui, W., Schulz, N. S., Baganoff, F. K., et al. 2001, ApJ, 548, 394

Del Santo, M., Bazzano, A., Zdziarski, A. A., et al. 2005, A\&A, 433, 613

Gallo, E., \& Fender, R. P. 2002, MNRAS, 337, 869

Gierlinski, M., Zdziarski, A. A., Done, C., et al. 1997, MNRAS, 288, 958

Hertz, P., \& Grindlay, J. E. 1984, ApJ, 278, 137

Jansen, F., Lumb, D., Altieri, B., et al. 2001, A\&A, 365, L1

Kuulkers, E., Chenevez, J., Wijnands, R., et al. 2013, ATel, 5332, 1

Lebrun, F., Leray, J. P., Lavocat, P., et al. 2003, A\&A, 411, L141

Lund, N., Budtz-Jørgensen, C., Westergaard, N. J., et al. 2003, A\&A, 411, L231

Magdziarz, P., \& Zdziarski, A. A. 1995, MNRAS, 273, 837

Martí, J., Mirabel, I. F., Chaty, S., \& Rodríguez, L. F. 2000, A\&A, 363, 184

Mirabel, I. F., Rodriguez, L. F., Cordier, B., Paul, J., \& Lebrun, F. 1992, Nature,

358,215

Natalucci, L., Tomsick, J. A., Bazzano, A., et al. 2014, ApJ, 780, 63
Poutanen, J., \& Svensson, R. 1996, ApJ, 470, 249

Remillard, R. A., \& McClintock, J. E. 2006, ARA\&A, 44, 49

Reynolds, M. T., \& Miller, J. M. 2010, ApJ, 716, 1431

Rybicki, G. B., \& Lightman, A. P. 1986, Radiative Processes in Astrophysics (New York: John Wiley and Sons)

Sakano, M., Imanishi, K., Tsujimoto, M., Koyama, K., \& Maeda, Y. 1999, ApJ, 520,316

Skinner, G. K., Willmore, A. P., Eyles, C. J., Bertram, D., \& Church, M. J. 1987, Nature, 330, 544

Skinner, G. K., Pan, H. C., Maisack, M., et al. 1991, A\&A, 252, 172

Smith, D. M., Heindl, W. A., \& Swank, J. H. 2002, ApJ, 578, L129

Strüder, L., Briel, U., Dennerl, K., et al. 2001, A\&A, 365, L18

Sunyaev, R., Churazov, E., Gilfanov, M., et al. 1991, ApJ, 383, L49

Titarchuk, L. 1994, ApJ, 434, 570

Turner, M. J. L., Abbey, A., Arnaud, M., et al. 2001, A\&A, 365, L27

Ubertini, P., Lebrun, F., Di Cocco, G., et al. 2003, A\&A, 411, L131

Wardziński, G., Zdziarski, A. A., Gierliński, M., et al. 2002, MNRAS, 337, 829

Winkler, C., Courvoisier, T. J.-L., Di Cocco, G., et al. 2003, A\&A, 411, L1

Zdziarski, A. A., Lubiński, P., \& Smith, D. A. 1999, MNRAS, 303, L11 